\title{
The Automorphism Group of the Lie Ring of Real Skew-Symmetric Matrices
}

\author{
Jinli Xu, ${ }^{1}$ Baodong Zheng, ${ }^{1}$ and Li Yang ${ }^{2}$ \\ ${ }^{1}$ Department of Mathematics, Harbin Institute of Technology, Harbin 150001, China \\ ${ }^{2}$ Department of Foundation, Harbin Finance University, Harbin 150030, China
}

Correspondence should be addressed to Baodong Zheng; zbd@hit.edu.cn

Received 11 May 2013; Accepted 17 July 2013

Academic Editor: T. Raja Sekhar

Copyright (c) 2013 Jinli Xu et al. This is an open access article distributed under the Creative Commons Attribution License, which permits unrestricted use, distribution, and reproduction in any medium, provided the original work is properly cited.

Denote by $\mathfrak{A}_{n}$ the set of all $n \times n$ skew-symmetric matrices over the field of real numbers, which forms a Lie ring under the usual matrix addition and the Lie multiplication as $[A, B]=A B-B A, A, B \in \mathfrak{A}_{n}$. In this paper, we characterize the automorphism group of the Lie ring $\mathfrak{A}_{n}$.

\section{Introduction and Main Result}

A Lie ring is defined as a nonassociative ring with multiplication that is anticommutative and satisfies the Jacobi identity. More specifically, we can define a Lie ring $L$ to be an abelian group with an operation [, ] that has the following properties:

(i) biadditive:

$$
[x+y, z]=[x, z]+[y, z], \quad[z, x+y]=[z, x]+[z, y],
$$

for all $x, y, z \in L$;

(ii) the Jacobi identity:

$$
[[x, y], z]+[[y, z], x]+[[z, x], y]=0,
$$

for all $x, y, z \in L$;

(iii) for all $x$ in $L$,

$$
[x, x]=0 .
$$

It is well known that a Lie algebra can be viewed as a Lie ring. So, the theory of Lie ring can be used in the theory of the Lie algebra. Recall that an automorphism of a Lie ring $(L,[]$, is a bijective map $\phi$ form $L$ onto itself such that $\phi(x+y)=$ $\phi(x)+\phi(y)$ and $\phi([x, y])=[\phi(x), \phi(y)]$ for all $x, y \in L$. There are a lot of papers that studied the automorphism groups of some fixed Lie rings (or, more for the Lie algebras), see [1-7].

Note that any associative ring $L$ can be made into a Lie ring $(L,+,[]$,$) by defining a bracket operator [x, y]=x y-$ $y x$. Let $\mathbb{R}$ be the real number field and denote by $\mathbb{R}^{\times}$the rest $\mathbb{R} \backslash\{0\}$. Let $M_{n}$ be the algebra of all $n \times n$ matrices over $\mathbb{R}$. We denote by $\mathfrak{A}_{n}$ the subset of $M_{n}$ consisting of all $n \times n$ skewsymmetric matrices, that is,

$$
\mathfrak{A}_{n}=\left\{\left[a_{i j}\right] \in M_{n}:\left[a_{i j}\right]^{t}=-\left[a_{i j}\right]\right\} .
$$

It is well known that the set $\boldsymbol{\mathfrak { A }}_{n}$ forms a Lie ring under the usual matrix addition and the Lie multiplication as $[A, B]=$ $A B-B A, A, B \in \mathfrak{A}_{n}$. In the same way, we know that $M_{n}$ or $T_{n}$ (the set of all $n \times n$ upper triangular matrices) as well as forms a Lie ring.

Hua [8] gives the form of any automorphism of the Lie ring $M_{n}$ over a skew field by using the fundamental theorem of geometry of matrices; Dolinar [1] studies the automorphism of the Lie ring of triangular matrices $T_{n}$ over any field. Jacobson [9] considers the Lie algebra $\mathfrak{A}_{n}$ over any algebraically closed field; he gives the form of the automorphism of the Lie algebra $\mathfrak{A}_{n}$ for the case $n \geq 5$ if $n$ is odd or $n \geq 10$ if $n$ is even. Now, let us see a general result on isomorphism of some Lie rings as follows.

Proposition 1 (see $[10,11])$. Let $A^{\prime}$ and $A$ be prime rings with involutions of the first kind and of characteristic not 2. 
Let $K^{\prime}$ and $K$ denote, respectively, the skew elements of $A^{\prime}$ and $A$. Assume that the dimension of the central closure of $A^{\prime}$ over $C_{A^{\prime}}$ is different from $1,4,9,16,25$, and 64. Then, any Lie isomorphism $\theta$ of $K^{\prime}$ onto $K$ can be extended uniquely to an associative isomorphism of $\left\langle K^{\prime}\right\rangle$ onto $\langle K\rangle$, the associative subrings generated by $K^{\prime}$ and $K$, respectively.

Note that the Lie ring $\mathfrak{A}_{n}$ is a particular class of the previous setting of skew elements of $M_{n}$. So, the previous proposition in fact partially solved the problem to characterize the automorphism group of $\mathfrak{A}_{n}$. However, the problem is still open when $n$ takes any positive integer.

The purpose of this paper is to characterize Aut $\mathfrak{A}_{n}$, the automorphism group of the Lie ring $\mathfrak{A}_{n}$, for $n \geq 2$. Our main result is the following.

Theorem 2. Suppose that $n \geq 2$ is an integer, then $\phi \in$ Aut $\mathfrak{A}_{n}$ if and only if there is a real orthogonal matrix $Q$ such that

$$
\phi(X)=Q X Q^{t}, \quad \forall X \in \mathfrak{\Re}_{n} .
$$

Further, one has Aut $\mathfrak{\Re}_{n} \cong O_{n}(R)$, where $O_{n}(R)$ is the real orthogonal group.

\section{Preliminary Results}

Now, let us start this section by denoting some notations. Denote by $[n / 2]$ the maximal integer number no more than $n / 2$. Let $E_{i j}^{(n)}$ be the $n \times n$ matrix which has 1 in the $(i, j)$ entry and is 0 elsewhere. Set $D=E_{12}^{(2)}+E_{21}^{(2)}$, and denote by $I_{n}=\sum_{i=1}^{n} E_{i i}^{(n)}$ the $n \times n$ identity matrix. Note that the notation $I_{0}$ means that the matrix vanished. Let $J=E_{11}^{(2)}-E_{22}^{(2)}$, $K=E_{12}^{(2)}-E_{21}^{(2)}$.

Suppose that $\mathfrak{S} \subset \mathfrak{A}_{n}$. We call $\mathfrak{S}$ to be commutative if $[x, \mathfrak{S}]=0$, for all $x \in \mathfrak{S}$, and call $\mathfrak{S}$ to be maximal commutative if $\mathbb{\mathfrak { S }}$ is not only commutative but $[y, \mathfrak{S}] \neq 0$, for all $y \notin \mathfrak{S}$. Clearly, the maximal commutative subset is a subring of $\mathfrak{A}_{n}$. Suppose that $A \in \mathfrak{\mathfrak { A }}_{n}$. Set

$$
\mathfrak{C}(A):=\left\{X \in \mathfrak{\mathcal { U }}_{n}:[A, X]=0\right\} .
$$

We denote by $A \oplus B$ and $A \otimes B$ the direct sum and the Kronecker product of $A$ and $B$, respectively.

Definition 3. A matrix $A \in \mathfrak{A}_{n}$ is called regular if it satisfies the following conditions: (i) when $n$ is an even number, there is an orthogonal matrix $Q$, and the real numbers $\varepsilon_{1}, \ldots, \varepsilon_{n / 2} \in$ $\mathbb{R}$ with different absolute values, such that

$$
A=Q \operatorname{diag}\left(\varepsilon_{1} K, \ldots, \varepsilon_{n / 2} K\right) Q^{t} .
$$

(ii) When $n$ is an odd number, there is an orthogonal matrix $Q$, and the nonzero real numbers $\varepsilon_{1}, \ldots, \varepsilon_{[n / 2]} \in \mathbb{R}$ with different absolute values, such that

$$
A=Q \operatorname{diag}\left(\varepsilon_{1} K, \ldots, \varepsilon_{[n / 2]} K \oplus 0\right) Q^{t} .
$$

Now, a subring $\mathfrak{h}$ of $\mathfrak{A}_{n}$ is called a regular subring if $\mathfrak{h}$ is maximal commutative and there is a regular matrix in $\mathfrak{h}$.

For $x \in \mathfrak{A}_{n}$, denote $\mathfrak{C}(x)=\left\{A \in \mathfrak{A}_{n}:[A, x]=0\right\}$.
Lemma 4 (see $[12,2.5 .14])$. Suppose that $A \in \mathfrak{A}_{n}$. Then, there are an orthogonal matrix $Q$ and real numbers $a_{1}, \ldots, a_{[n / 2]}$ such that

$$
A=Q \operatorname{diag}\left(a_{1} K \oplus \cdots \oplus a_{[n / 2]} K \oplus 0\right) Q^{t} .
$$

Lemma 5 (see $[13,14]$ ). Let $\mathbb{F}$ be any field, and let $K_{n}(\mathbb{F})$ denote the space of all $n \times n$ alternate matrices over $\mathbb{F}$. Then, $\phi$ is an additive surjective mapping of $K_{n}(\mathbb{F})(n \geq 2)$ to itself that preserves rank 2 matrices if and only if $\phi$ is of the following forms:

(i) $n \geq 4, \phi\left(\left(a_{i j}\right)\right)=\alpha P^{t}\left(f\left(a_{i j}\right)\right) P$, for all $\left(a_{i j}\right) \in K_{n}(\mathbb{F})$, where $\alpha \in \mathbb{F} \backslash\{0\}, P$ is an $n \times n$ invertible matrix, and $f$ is a field automorphism of $\mathbb{F}$;

(ii) when $n=4$, $\phi$ is of the form

$$
\phi\left(\left(a_{i j}\right)\right)=\alpha P^{t}\left(f\left(a_{i j}\right)\right)^{*} P, \quad \forall\left(a_{i j}\right) \in K_{4}(\mathbb{F}),
$$

where $\alpha, P$, and $f$ have the same meaning as before, and $\left(a_{i j}\right) \mapsto\left(a_{i j}\right)^{*}$ is either the identity map or the map:

$$
\left[\begin{array}{cccc}
0 & a_{12} & a_{13} & a_{14} \\
-a_{12} & 0 & a_{23} & a_{24} \\
-a_{13} & -a_{23} & 0 & a_{34} \\
-a_{14} & -a_{24} & -a_{34} & 0
\end{array}\right] \longmapsto\left[\begin{array}{cccc}
0 & a_{12} & a_{13} & a_{23} \\
-a_{12} & 0 & a_{14} & a_{24} \\
-a_{13} & -a_{14} & 0 & a_{34} \\
-a_{23} & -a_{24} & -a_{34} & 0
\end{array}\right]
$$

In the next text, we always assume that $\phi \in \operatorname{Aut} \mathfrak{A}_{n}$ is arbitrary.

Lemma 6. Suppose that $\mathfrak{h}$ is a regular subring of $\mathfrak{A}_{n}$. Then, there is an orthogonal matrix $Q$ and maps $\eta_{i}: \mathfrak{A}_{n} \rightarrow \mathbb{R}, i=$ $1, \ldots,[n / 2]$ such that

$$
\mathrm{QXQ}^{t}=\eta_{1}(X) K \oplus \cdots \oplus \eta_{[n / 2]}(X) K \oplus 0, \quad \forall X \in \mathfrak{h} .
$$

Proof. For every $X \in \mathfrak{h}$, note that $Q X Q^{t}$ is commutative with every regular matrix in $\mathfrak{h}$. So, one can obtain the conclusion by Lemma 4 .

Corollary 7. Suppose that $\mathfrak{h}$ is a regular subring of $\mathfrak{A}_{n}$, and $H \in \mathfrak{h}$ is a regular matrix. Then,

$$
X \in \mathfrak{h} \Longleftrightarrow[X, H]=0 .
$$

Lemma 8. Suppose that $\mathfrak{h}_{1}, \mathfrak{h}_{2}$ are both regular subrings of $\mathfrak{A}_{n}$, and that there is a regular matrix $H \in \mathfrak{h}_{1} \cap \mathfrak{h}_{2}$. Then, $\mathfrak{h}_{1}=\mathfrak{h}_{2}$.

Proof. Note that a regular subring is maximal; the conclusion follows by Corollary 7.

Lemma 9. Both maps $\phi$ and $\phi^{-1}$ preserve the regular subring. Expressly, for $H \in \mathfrak{A}_{n}$, one has that $H$ is a regular matrix if and only if $\phi(H)$ is so.

Proof. Take any regular subring $\mathfrak{h}$ of $\mathfrak{A}_{n}$ and a regular matrix $H \in \mathfrak{h}$. By Lemma 6, we can assume that $Q \phi(H) Q^{t}=\varepsilon_{1} K \oplus$ $\cdots \oplus \varepsilon_{[n / 2]} K \oplus 0$, where $Q$ is an orthogonal matrix. 
Suppose that $A \in \mathfrak{A}_{n}$ satisfying $Q \phi(A) Q^{t}=1 K \oplus 2 K \oplus$ $\cdots \oplus[n / 2] K \oplus 0$. Then, $\phi(A)$ is a regular matrix in $\mathfrak{A}_{n}$. Since $[\phi(H), \phi(A)]=0,[H, A]=0$. This means that $A \in \mathfrak{h}$. Let $\mathfrak{h}_{1}$ be a regular subring containing $\phi(A)$. By Lemma 8 , we only need to prove that $\phi(\mathfrak{h})=\mathfrak{h}_{1}$. Take any $\phi(X) \in \mathfrak{h}_{1}$. Then, we see by Lemma 6 that there are $x_{1}, \ldots, x_{[n / 2]} \in \mathbb{R}$ such that

$$
\mathrm{Q} \phi(X) Q^{t}=x_{1} K \oplus \cdots \oplus x_{[n / 2]} K \oplus 0 .
$$

So, $[\phi(X), \phi(H)]=0$, and $[X, H]=0$. Hence, $X \in \mathfrak{h}$. This shows that $\mathfrak{h}_{1} \subset \phi(\mathfrak{h})$. Note that $\mathfrak{h}_{1}$ is maximal, so we obtain that $\phi(\mathfrak{h})=\mathfrak{h}_{1}$.

Now, we prove that $\phi$ preserves the regular matrix. Otherwise, suppose that $\phi(H)$ is not a regular matrix, then we will get a contradiction. By the definition, we see that one of the following cases holds.

Case 1. $n$ is odd and there is $\varepsilon_{i}=0$.

Case 2. There is some $\varepsilon_{i} \in\left\{ \pm \varepsilon_{j}\right\}$.

If Case 1 happens, we assume without loss the generality that $\varepsilon_{1}=0$. We take $X \in \mathfrak{A}_{n}$ such that

$$
Q \phi(X) Q^{t}=E_{1 n}^{(n)}-E_{n 1}^{(n)} .
$$

If Case 2 happens, we assume without loss the generality that $\varepsilon_{2} \in\left\{ \pm \varepsilon_{1}\right\}$. When $\varepsilon_{1}=\varepsilon_{2}$, we take $X \in \mathfrak{A}_{n}$ such that

$$
Q \phi(X) Q^{t}=K \otimes I_{2} \oplus 0 .
$$

When $\varepsilon_{1}=-\varepsilon_{2}$, we take $X \in \mathfrak{A}_{n}$ such that

$$
Q \phi(X) Q^{t}=K \otimes J \oplus 0 .
$$

On one hand, it is clear that $[\phi(X), \phi(A)] \neq 0$, so we have $\phi(X) \notin \mathfrak{h}_{1}$. On the other hand, $[\phi(X), \phi(H)]=0$; hence, $[X, H]=0$. Thus, $X \in \mathfrak{h}$, and so $\phi(X) \in \phi(\mathfrak{h})=\mathfrak{h}_{1}$; this is impossible. Note that $\phi$ is an automorphism; we see that $\phi^{-1}$ also preserves the regular matrix. The proof is completed.

Lemma 10. Suppose that $n \geq 5$ and $A, B \in \mathfrak{A}_{n}$. If $\operatorname{rank} A=2$ and $B \notin \mathbb{R} A$, then there is $C \in \mathfrak{A}_{n}$ such that

$$
[A, C]=0, \quad[B, C] \neq 0 .
$$

Proof. We can assume without loss the generality by Lemma 4 that $A=a K \oplus 0, a \neq 0$. Hence, we have $\mathfrak{C}(A)=$ $0 \oplus \mathfrak{A}_{n-2}$. If any matrix $C$ cannot satisfy the conclusion, then one has $\left[B, 0 \oplus \mathfrak{A}_{n-2}\right]=0$. Note that $n \geq 5$, so we have $n-2 \geq 3$. This implies that $B \in \mathbb{R} K \oplus 0$, which contradicts with $B \notin \mathbb{R} A$.

Lemma 11. Let $A \in \mathfrak{A}_{n}$. Then, $\phi(\mathfrak{C}(A))=\mathfrak{C}(\phi(A))$.

Proof. As $[A, \mathfrak{C}(A)]=0$, we deduce that $[\phi(A), \phi(\mathfrak{C}(A))]=$ 0 . Farther, we have $\phi(\mathfrak{C}(A)) \subset \mathfrak{C}(\phi(A))$. The desired result follows from the following:

$$
\begin{aligned}
& \mathfrak{C}(\phi(A))=\left(\phi \phi^{-1}\right) \mathfrak{C}(\phi(A))=\phi\left(\phi^{-1}(\mathfrak{C}(\phi(A)))\right) \\
& \subset \phi\left(\mathfrak{C}\left(\phi^{-1}(\phi(A))\right)\right)=\phi(\mathfrak{C}(A)) .
\end{aligned}
$$

Lemma 12. Suppose that $A \in \mathfrak{A}_{4}$ is not a regular matrix. Then, $\phi(\mathbb{R} A)=\mathbb{R} \phi(A)$.

Proof. It follows from Lemma 6 that there is an orthogonal matrix $Q$ such that

$$
\phi(r A)=Q\left(\varepsilon_{1}(r) K \oplus \varepsilon_{2}(r) K\right) Q^{t}, \quad \forall r \in \mathbb{R} .
$$

Since $A$ is not a regular matrix and so is $r A$, we see that $\varepsilon_{2}(r) \in\left\{ \pm \varepsilon_{1}(r)\right\}$, for all $r \in \mathbb{R}$. If $\varepsilon_{1}(1)=\varepsilon_{2}(1)$, then we will see that $\varepsilon_{1}=\varepsilon_{2}$. Otherwise, there is $r_{0} \in \mathbb{R}^{\times}$such that $\varepsilon_{1}\left(r_{0}\right)=$ $-\varepsilon_{2}\left(r_{0}\right)$, and so $Q\left(K \otimes I_{2}\right) Q^{t} \in \mathfrak{C}(\phi(A))$. But we know that $Q\left(K \otimes I_{2}\right) Q^{t} \notin \mathfrak{C}\left(\phi\left(r_{0} A\right)\right)$, this, together with Lemma 11, gives that

$$
\mathfrak{C}(\phi(A))=\phi(\mathfrak{C}(A))=\phi\left(\mathfrak{C}\left(r_{0} A\right)\right)=\mathfrak{C}\left(\phi\left(r_{0} A\right)\right) .
$$

This is impossible. Similarly, we can show that if $\varepsilon_{1}(1)=$ $-\varepsilon_{2}(1)$, then $\varepsilon_{1}=-\varepsilon_{2}$, and then we get the conclusion.

Lemma 13. Suppose that $n \geq 5$ and $A \in \mathfrak{A}_{n}$ such that $\operatorname{rank} A=2$. Then, $\phi(\mathbb{R} A)=\mathbb{R} \phi(A)$.

Proof. If there is $B \notin \mathbb{R} A$ such that $\phi(B) \in \mathbb{R} \phi(A)$, then by Lemma 10 we can choose $C \in \mathfrak{A}_{n}$ such that $[A, C]=0$, $[B, C] \neq 0$. Thus, $[\phi(A), \phi(C)]=0 ;[\phi(B), \phi(C)] \neq 0$. But we see that $\phi(B) \in \mathbb{R} \phi(A)$; this is impossible. Furthermore,

$$
\mathbb{R} \phi(A) \subset \phi(\mathbb{R} A) .
$$

For any nonzero real number $r$, we replace $A$ by $r A$ in the previous equation. It follows that

$$
\mathbb{R} \phi(\mathbb{R} A) \subset \phi(\mathbb{R} A) \subset \mathbb{R} \phi(\mathbb{R} A),
$$

that is, $\phi(\mathbb{R} A)=\mathbb{R} \phi(\mathbb{R} A)$. Note that $\phi$ is additive. So, $\phi(\mathbb{R} A)$ is a subspace.

Suppose that

$$
\phi(r A)=Q \operatorname{diag}\left(\varepsilon_{1}(r) K \oplus \cdots \oplus \varepsilon_{[n / 2]}(r) K \oplus 0\right) Q^{t},
$$

where $Q$ is an orthogonal matrix, $\varepsilon_{i}: \mathbb{R} \rightarrow \mathbb{R}, i=1, \ldots,[n / 2]$. We first prove the following.

Assertion. If there is an index $i_{0}$ such that $\varepsilon_{i_{0}}(r) \neq 0$, for all $r \epsilon$ $\mathbb{R}^{\times}$, then $\phi(\mathbb{R} A) \subset \mathbb{R} \phi(A)$.

In fact, for any given $r \in \mathbb{R}^{\times}$, suppose that $\varepsilon_{i_{0}}(r)=\rho \varepsilon_{i_{0}}(1)$, then $\rho \neq 0$. Now, we assume that the assertion is not true; then there is some index $s$ such that $\varepsilon_{s}(r) \neq \rho \varepsilon_{s}(1)$. Then, we see by $\phi(A) \in \phi(\mathbb{R} A), \phi(r A) \in \phi(\mathbb{R} A)$, and the fact $\phi(\mathbb{R} A)$ is a space that $\rho \phi(A)-\phi(r A) \in \phi(\mathbb{R} A)$. This tells us that there is some $c \in \mathbb{R}$ such that $\rho \phi(A)-\phi(r A)=\phi(c A)$. Thus, $\varepsilon_{s}(c)=\rho \varepsilon_{s}(1)-\varepsilon_{s}(r) \neq 0$, and so we have $c \neq 0$. But we know that $\varepsilon_{i_{0}}(c)=\rho \varepsilon_{i_{0}}(1)-\varepsilon_{i_{0}}(r)=0$, which contradicts with the conditions of the assertion. This gives that $\phi(r A)=\rho \phi(A)$. The assertion is proved.

As $A$ is not a regular matrix, one has that $\phi(r A)$ is not a regular matrix too. Next, the proof of the lemma is divided into the following cases with respect to $n$.

Case 1. When $n$ is odd, note that $A \neq 0$, so we can assume without loss the generality that $\varepsilon_{1}(1) \neq 0$. If for some 
$r_{0} \in \mathbb{R}^{\times}$such that $\varepsilon_{1}\left(r_{0}\right)=0$, then it follows by Lemma 11 that $Q\left(E_{1 n}^{(n)}-E_{n 1}^{(n)}\right) Q^{t} \in \mathfrak{E}\left(\phi\left(r_{0} A\right)\right)=\mathfrak{C}(\phi(A))$, which is a contradiction. Now, the lemma follows by using the previous assertion for the index $i_{0}=1$.

Case 2. When $n$ is even, assume without loss of the generality that $\varepsilon_{1}(1) \in\left\{ \pm \varepsilon_{2}(1)\right\}$. If $\varepsilon_{1}(1)=\varepsilon_{2}(1)$, then $\varepsilon_{1}=\varepsilon_{2}$. In fact, if there is some $r_{0} \in \mathbb{R}^{\times}$such that $\varepsilon_{1}\left(r_{0}\right) \neq \varepsilon_{2}\left(r_{0}\right)$, then one has by Lemma 11 that $Q\left(K \otimes I_{2} \oplus 0\right) Q^{t} \in \mathfrak{S}(\phi(A))=\mathfrak{S}\left(\phi\left(r_{0} A\right)\right)$ is a contradiction. If $\varepsilon_{1}(1)=-\varepsilon_{2}(1)$, then $\varepsilon_{1}=-\varepsilon_{2}$. In fact, if there is some $r_{0} \in \mathbb{R}^{\times}$such that $\varepsilon_{1}\left(r_{0}\right) \neq-\varepsilon_{2}\left(r_{0}\right)$, then we see by Lemma 11 that $Q(K \otimes J \oplus 0) Q^{t} \in \mathfrak{S}(\phi(A))=\mathfrak{S}\left(\phi\left(r_{0} A\right)\right)$; this is impossible.

When $\varepsilon_{1}(1) \neq 0$, if there is $r_{0} \in \mathbb{R}^{\times}$such that $\varepsilon_{1}\left(r_{0}\right)=0$, then $\varepsilon_{2}\left(r_{0}\right)=0$. Thus, $\mathfrak{A}_{4} \oplus 0 \subset \mathfrak{C}\left(\phi\left(r_{0} A\right)\right)=\mathfrak{S}(\phi(A))$, which is a contradiction. Now, we get the lemma by using the previous assertion for the index $i_{0}=1$.

When $\varepsilon_{1}(1)=0$, if there is $r_{0} \in \mathbb{R}^{\times}$such that $\varepsilon_{1}\left(r_{0}\right) \neq 0$, then since $\varepsilon_{2}(1)=\varepsilon_{1}(1)=0, \mathfrak{A}_{4} \oplus 0 \subset \mathfrak{C}(\phi(A))=\mathfrak{C}\left(\phi\left(r_{0} A\right)\right)$. This is absurd. As $A \neq 0$, it is clear that $n \geq 6$. Hence, we can assume without loss of the generality that $\varepsilon_{3}(1) \neq 0$. If for some $r_{0} \in \mathbb{R}^{\times}$such that $\varepsilon_{3}\left(r_{0}\right)=0$, then we have by Lemma 11 that

$$
Q\left(\left(E_{15}^{(5)}-E_{51}^{(5)}\right) \oplus 0\right) Q^{t} \in \mathfrak{S}\left(\phi\left(r_{0} A\right)\right)=\mathfrak{S}(\phi(A))
$$

which is a contradiction. The lemma can be shown by using the previous assertion for the index $i_{0}=3$.

Corollary 14. Suppose that $n \geq 5$ and $W \leq \mathfrak{A}_{n}$ is a subspace with bases which are formed by rank 2 matrices. Then, we have

$$
\operatorname{dim} W=\operatorname{dim} \phi(W) .
$$

Proof. Suppose that rank 2 matrices $e_{1}, \ldots, e_{s}$ form bases of $W$. Then,

$$
\mathbb{R} e_{1} \oplus \cdots \oplus \mathbb{R} e_{s}=W
$$

It follows immediately that

$$
\phi\left(\mathbb{R} e_{1}\right)+\cdots+\phi\left(\mathbb{R} e_{s}\right)=\phi(W) .
$$

If there is $i$ such that $\phi\left(\mathbb{R} e_{i}\right) \cap \sum_{j \neq i} \phi\left(\mathbb{R} e_{j}\right) \neq 0$, then we can choose $\lambda_{1}, \ldots, \lambda_{s} \in \mathbb{R}$, not all zero, such that $\phi\left(\lambda_{i} e_{i}\right)=$ $\sum_{j \neq i} \phi\left(\lambda_{j} e_{j}\right)$, which is absurd. We see by Lemma 13 that $\phi\left(\mathbb{R} e_{i}\right)=\mathbb{R} \phi\left(e_{i}\right)$, for all $i=1, \ldots, s$. Thus,

$$
\mathbb{R} \phi\left(e_{1}\right) \oplus \cdots \oplus \mathbb{R} \phi\left(e_{s}\right)=\phi(W) .
$$

The proof is completed.

Lemma 15. Suppose that $n \geq 5$ and $A \in \mathfrak{A}_{n}$ is of rank 2. Then,

$$
\operatorname{dim} \mathfrak{S}(\phi(A))=\frac{1}{2}(n-2)(n-3)+1 .
$$

Proof. Note that $\operatorname{dim} \mathfrak{E}(A)=\operatorname{dim} \mathfrak{A}_{n-2}+1=(1 / 2)(n-$ $2)(n-3)+1$ and $\mathfrak{C}(A)$ has bases which are formed by rank 2 matrices. This, together with Corollary 14, proves the conclusion.
Lemma 16. Suppose that $a_{1}, \ldots, a_{s}$ are positive real numbers, which are different from one another. Let $x=a_{1} I_{n_{1}} \otimes K \oplus$ $-a_{1} I_{n_{-1}} \otimes K \oplus \cdots \oplus a_{s} I_{n_{s}} \otimes K \oplus-a_{s} I_{n_{-s}} \otimes K \oplus 0$. Then, we have

$$
\begin{aligned}
\operatorname{dim} \mathfrak{C}(x)= & \left(\Sigma_{i}\left(n_{i}+n_{-i}\right)\right)^{2}+2^{-1} \\
& \times\left(n-2 \Sigma_{i}\left(n_{i}+n_{-i}\right)\right)\left(n-2 \Sigma_{i}\left(n_{i}+n_{-i}\right)-1\right) .
\end{aligned}
$$

In particular, if we let $d=\Sigma_{i}\left(n_{i}+n_{-i}\right)$, then

$$
\operatorname{dim} \mathfrak{C}(x) \leq d^{2}+2^{-1}(n-2 d)(n-2 d-1),
$$

and the equation holds if and only if $s=1$.

Proof. It follows by a direct computation.

Lemma 17. Suppose that $\phi \in$ Aut $\mathfrak{A}_{n}$ preserves the rank 2 matrix subset of $\mathfrak{A}_{n}$. Then, there is a real orthogonal matrix Q such that

$$
\phi(X)=Q X Q^{t}, \quad \forall X \in \mathfrak{A}_{n} .
$$

Proof. The proof under the case $n=2$ is obvious. It is not difficult to see that, if $n=3$, then a surjective map preserving rank 2 matrices still is of the form (i) of Lemma 5. Next, we assume that $n \geq 3$ and assume that $\phi$ has the form (i) of Lemma 5. For distinct $i, j, k$, it is clear that

$$
\begin{aligned}
& {\left[E_{i j}-E_{j i}, E_{i k}-E_{k i}\right]=E_{k j}-E_{j k},} \\
& {\left[E_{i j}-E_{j i}, E_{j k}-E_{k j}\right]=E_{i k}-E_{k i} .}
\end{aligned}
$$

Consider the image of $\phi$; then, it follows by the form (i) of Lemma 5 that

$$
\begin{aligned}
& \alpha\left(\left(E_{i j}-E_{j i}\right) P^{t} P\left(E_{i k}-E_{k i}\right)\right. \\
& \left.\quad-\left(E_{i k}-E_{k i}\right) P^{t} P\left(E_{i j}-E_{j i}\right)\right)=E_{k j}-E_{j k}, \\
& \alpha\left(\left(E_{i j}-E_{j i}\right) P^{t} P\left(E_{j k}-E_{k j}\right)\right. \\
& \left.\quad-\left(E_{j k}-E_{k j}\right) P^{t} P\left(E_{i j}-E_{j i}\right)\right)=E_{i k}-E_{k i} .
\end{aligned}
$$

Hence, by a direct computation and the arbitrariness of $i$, $j, k$, it follows that $P^{t} P=\alpha^{-1} I_{n}$. Clearly, $\alpha>0$. Note that $\mathbb{R}$ is the field of real numbers, so we have $f=1$. Let $Q=\sqrt{\alpha} P$; then, the conclusion is obtained.

When $n=4$ and $\phi$ is of the form (ii) of Lemma 5 , then we let $i=1, j=2$, and $k=3$. Thus, we have by taking the images under $\phi$ for the previous two equations that $1=0$, which is a contradiction. So, the form (ii) of Lemma 5 does not occur.

\section{The Proof of the Main Result}

The proof of the main theorem is divided into the following three propositions.

Proposition 18. Suppose that $n=2$ or 3 and $\phi \in$ Aut $\mathfrak{A}_{n}$. Then, there is an orthogonal matrix $Q$ such that

$$
\phi(X)=Q X Q^{t}, \quad \forall X \in \mathfrak{A}_{n} .
$$


Proof. Since $\phi$ is bijective, $\phi$ preserves the rank 2 matrices of $\mathfrak{U}_{2}$ or $\mathfrak{A}_{3}$. If $n=2$, the conclusion is clear. If $n=3$, then we also can get the conclusion by Lemma 17 .

Proposition 19. Suppose that $\phi \in$ Aut $\mathfrak{A}_{4}$. Then, there is an orthogonal matrix $Q$ such that

$$
\phi(X)=Q X Q^{t}, \quad \forall X \in \mathfrak{A}_{4}
$$

Proof. It is clear that $[K, D]=2 J,[J, K]=2 D$, and $[J, D]=$ $2 K$. Note that $K \oplus 0$ is regular, so we can assume that $\phi(K \oplus 0)=$ $Q(a K \oplus b K) Q^{t}$, where $a \neq \pm b$ and $Q$ is an orthogonal matrix. Without loss of generality, one can assume that

$$
\phi(K \oplus 0)=a K \oplus b K .
$$

Since the regular subring containing the nonregular matrix $\phi\left(I_{2} \otimes K\right)$ is determined by $\phi(K \oplus 0)$, there are $c \in \mathbb{R}^{\times}$ and $\varepsilon \in\{ \pm 1\}$ such that

$$
\phi\left(I_{2} \otimes K\right)=c(K \oplus \varepsilon K)
$$

Therefore,

$$
\phi(0 \oplus K)=\phi\left(I_{2} \otimes K\right)-\phi(K \oplus 0)=(c-a) K \oplus(\varepsilon c-b) K .
$$

Suppose that

$$
\phi\left(K \otimes I_{2}\right)=\left[\begin{array}{cc}
X_{1} & Y_{1} \\
-Y_{1}^{t} & Z_{1}
\end{array}\right]
$$

where $X_{1}$ is a $2 \times 2$ matrix. It follows by $\left[I_{2} \otimes K, K \otimes I_{2}\right]=0$ that

$$
\left[c(K \oplus \varepsilon K), \phi\left(K \otimes I_{2}\right)\right]=0
$$

So, we have $K X=X K, K Z=Z K, K Y_{1}=\varepsilon Y_{1} K$, and $K Y^{t}=\varepsilon Y^{t} K$. Note that

$$
\left[K \oplus 0,\left[K \oplus 0, K \otimes I_{2}\right]\right]=-K \otimes I_{2}
$$

Thus,

$$
\left[\left[\begin{array}{cc}
a K & 0 \\
0 & b K
\end{array}\right],\left[\left[\begin{array}{cc}
a K & 0 \\
0 & b K
\end{array}\right],\left[\begin{array}{cc}
X_{1} & Y_{1} \\
-Y_{1}^{t} & Z_{1}
\end{array}\right]\right]\right]=-\left[\begin{array}{cc}
X_{1} & Y_{1} \\
-Y_{1}^{t} & Z_{1}
\end{array}\right] .
$$

This, together with $K Y_{1}=\varepsilon Y_{1} K$, gives that $(a-\varepsilon b)^{2}=1$, $X_{1}=0, Z_{1}=0$. We deduce that

$$
\phi\left(K \otimes I_{2}\right)=\left[\begin{array}{cc}
0 & Y_{1} \\
-Y_{1}^{t} & 0
\end{array}\right]
$$

Similarly, we see by $\left[I_{2} \otimes K, D \otimes K\right]=0$ and $[K \oplus 0,[K \oplus$ $0, D \otimes K]]=-D \otimes K$ that

$$
\phi(D \otimes K)=\left[\begin{array}{cc}
0 & Y_{2} \\
-Y_{2}^{t} & 0
\end{array}\right]
$$

where $Y_{2} K=\varepsilon K Y_{2}$. We also have by $\left[K \otimes I_{2}, D \otimes K\right]=2 J \otimes K$ that

$$
\begin{aligned}
\phi(J \otimes K) & =2^{-1}\left[\left[\begin{array}{cc}
0 & Y_{1} \\
-Y_{1}^{t} & 0
\end{array}\right],\left[\begin{array}{cc}
0 & Y_{2} \\
-Y_{2}^{t} & 0
\end{array}\right]\right] \\
& =2^{-1}\left[\begin{array}{cc}
Y_{2} Y_{1}^{t}-Y_{1} Y_{2}^{t} & 0 \\
0 & Y_{2}^{t} Y_{1}-Y_{1}^{t} Y_{2}
\end{array}\right] .
\end{aligned}
$$

Note that $Y_{i} K=\varepsilon K Y_{i}$, so we can assume that

$$
Y_{1}=\left[\begin{array}{cc}
y_{1} & y_{2} \\
-\varepsilon y_{2} & \varepsilon y_{1}
\end{array}\right], \quad Y_{2}=\left[\begin{array}{cc}
y_{3} & y_{4} \\
-\varepsilon y_{4} & \varepsilon y_{3}
\end{array}\right] \text {. }
$$

Further,

$$
\begin{gathered}
Y_{2} Y_{1}^{t}-Y_{1} Y_{2}^{t}=2 \varepsilon\left(y_{1} y_{4}-y_{2} y_{3}\right) K, \\
Y_{2}^{t} Y_{1}-Y_{1}^{t} Y_{2}=-2\left(y_{1} y_{4}-y_{2} y_{3}\right) K, \\
\phi(J \otimes K)=\left(y_{1} y_{4}-y_{2} y_{3}\right)(\varepsilon K \oplus-K) .
\end{gathered}
$$

On the other hand, we know by $J \otimes K=K \oplus 0-0 \oplus K$ that

$$
\phi(J \otimes K)=(2 a-c) K \oplus(2 b-\varepsilon c) K .
$$

By a direct computation with (49) and (50), we have $2 a-$ $c=c-2 \varepsilon b=\varepsilon\left(y_{1} y_{4}-y_{2} y_{3}\right)$ and then $a+\varepsilon b=c$. Noting that $(a-\varepsilon b)^{2}=1$, we can assume without loss of the generality that $a-\varepsilon b=1$ (for the case $a-\varepsilon b=-1$, the proof is similar). We deduce that

$$
\phi(J \otimes K)=J \otimes K
$$

If $\varepsilon=-1$, then $\phi\left(I_{2} \otimes K\right)=c(K \oplus-K) \in \mathbb{R} \phi(J \otimes K)$, which contradicts Lemma 12 . This tells us that $\varepsilon=1$.

Again by $\left[K \oplus 0, K \otimes I_{2}\right]=D \otimes K$, we get $Y_{2}=K Y_{1}$.

Suppose that

$$
\phi(K \otimes J)=\left[\begin{array}{cc}
X_{3} & Y_{3} \\
-Y_{3}^{t} & Z_{3}
\end{array}\right] .
$$

It follows by $[J \otimes K, K \otimes J]=0$ that

$$
\left[J \otimes K,\left[\begin{array}{cc}
X_{3} & Y_{3} \\
-Y_{3}^{t} & Z_{3}
\end{array}\right]\right]=0
$$

Therefore, we have $K Y_{3}+Y_{3} K=0$. Note that $\left[I_{2} \otimes K,\left[I_{2} \otimes\right.\right.$ $K, K \otimes J]]=-4 K \otimes J$. This tells us that $c^{2}=1$. We can assume without loss of the generality that $c=1$. Then, we get that $a=1$ and $b=0$. Thus,

$$
\phi(K \oplus 0)=K \oplus 0 .
$$

For any but fixed $r \in \mathbb{R}^{\times}$, we assert that $\phi(r K \oplus 0) \in \mathbb{R} K \oplus 0$. In fact, firstly, by Lemma 12 , we can assume that $\phi\left(r I_{2} \otimes\right.$ $K)=s I_{2} \otimes K, \phi(r K \oplus 0)=u K \oplus v K$. So, we have that $\phi(0 \oplus r K)=$ $(s-u) K \oplus(s-v) K$.

Secondly, noting that $\left[I_{2} \otimes K, r K \otimes I_{2}\right]=0$ and $[r K \oplus 0,[K \oplus$ $\left.\left.0, K \otimes I_{2}\right]\right]=-r K \otimes I_{2}$, we deduce that

$$
\phi\left(r K \otimes I_{2}\right)=(u-v) \phi\left(K \otimes I_{2}\right) .
$$


Furthermore, we see by $r J \otimes K=(1 / 2)\left[r K \otimes I_{2},[K \oplus 0, K \otimes\right.$ $\left.\left.I_{2}\right]\right]$ and $r J \otimes K=r K \oplus 0-0 \oplus r K$ that

$$
\begin{gathered}
\phi(r J \otimes K)=(u-v) J \otimes K, \\
\phi(r J \otimes K)=(2 u-s) K \oplus(2 v-s) K .
\end{gathered}
$$

Thus, $(2 u-s)=u-v=(s-2 v)$. It follows that $u+v=s$.

Finally, due to $[r K \oplus 0,[K \oplus 0, K \otimes J]]=-r K \otimes J$ and $\left[r I_{2} \otimes K,\left[I_{2} \otimes K, K \otimes J\right]\right]=-4 r K \otimes J$, one can obtain that

$$
\phi(r K \otimes J)=s \phi(K \otimes J)=(u-v) \phi(K \otimes J) .
$$

This tells us that $u-v=s$, and so we have that $u=s, v=0$. In other words, $\phi(r K \oplus 0)=u K \oplus 0$, which proves the assertion. Now, we prove that $\phi$ preserves the set of rank 2 matrices on $\mathfrak{A}_{4}$. By applying Lemma 17 , we finish the proof.

Proposition 20. Suppose that $n \geq 5$ and $\phi \in$ Aut $\mathfrak{A}_{n}$. Then, there is an orthogonal matrix $Q$ such that

$$
\phi(X)=Q X Q^{t}, \quad \forall X \in \mathfrak{\Re}_{n} .
$$

Proof. Take any rank 2 matrix $A \in \mathfrak{A}_{n}$. By Lemma 4 , we can assume that

$$
\begin{aligned}
\phi(A)=Q( & a_{1} I_{n_{1}} \otimes K \oplus-a_{1} I_{n_{-1}} \otimes K \oplus \cdots \oplus a_{s} I_{n_{s}} \otimes K \oplus \\
\left.-a_{s} I_{n_{-s}} \otimes K \oplus 0\right) Q^{t} &
\end{aligned}
$$

Let $\Sigma_{i}\left(n_{i}+n_{-i}\right)=d$. Now, we assert that $d=1$ and so that the rank of $\phi(A)$ is 2 ; that is, we will assert that $\phi$ is a preserver of rank 2 on $\mathfrak{A}_{n}$; then, we can finish the proof by Lemma 17.

It follows by Lemmas 15 and 16 that

$$
\frac{1}{2}(n-2)(n-3)+1 \leq d^{2}+2^{-1}(n-2 d)(n-2 d-1) .
$$

Moreover, we see that $(d-1)(3 d-2 n+4) \geq 0$. Hence, we have either $d \leq 1$ or $d \geq 3^{-1}(2 n-4)$. The former means that $d=1$, as desired. If the latter holds, then it is clear that

$$
n \geq 2 d \geq 2 \cdot 3^{-1}(2 n-4)
$$

In this case, we deduce that $n \leq 8$ and $n \neq 7$. Hence, the remainder of the proof is the cases (i) $n=5, d=2$, (ii) $n=6$, $d=3$, and (iii) $n=8, d=4$.
Suppose that $B=Q(0 \oplus K \oplus 0) Q^{t}$. We consider the rank of $\phi(B)$.

When $\operatorname{rank} \phi(B)=2$, it is clear that there is an orthogonal matrix $P$ such that $\phi(A)=P\left(\varepsilon I_{p} \otimes K \oplus-\varepsilon I_{q} \otimes K \oplus 0\right) P^{t}$ and $\phi(B)=P(\eta K \oplus 0) P^{t}$. Without loss of the generality, we can assume that $p \neq 0$. Note that $\eta \neq 0$. If $\eta \neq-2 \varepsilon$, then one has $\varepsilon+\eta \neq-\varepsilon$. Let $C=Q\left(K \otimes I_{2} \oplus 0\right) Q^{t}$. As $[A+B, C]=0$, we can find a matrix $X \in \mathfrak{A}_{n-4}$ such that

$$
\phi(C)=P(0 \oplus X) P^{t} .
$$

If $\eta=-2 \varepsilon$, then $\varepsilon-\eta \neq-\varepsilon$. Let $C=Q(K \otimes J \oplus 0) Q^{t}$. Since $[A-B, C]=0$, there is a matrix $X \in \mathfrak{A}_{n-4}$ such that

$$
\phi(C)=P(0 \oplus X) P^{t} .
$$

Thanks to $[B,[B, C]]=-C$, we deduce $\phi(C)=0$, which is a contradiction.

When $\operatorname{rank} \phi(B) \neq 2$, then for the previous three cases of $n$ and $d$, one always has $\operatorname{rank} \phi(B)=\operatorname{rank} \phi(A)$. Note that $\phi(A)$ and $\phi(B)$ are in a common regular subring, and $s=1$. It follows by Lemma 6 that there is an orthogonal matrix $P$ such that $\phi(A)=P\left(\varepsilon_{1} K \oplus \cdots \oplus \varepsilon_{d} K \oplus 0\right) P^{t}$ and $\phi(B)=P\left(\eta_{1} K \oplus \cdots \oplus\right.$ $\left.\eta_{d} K \oplus 0\right) P^{t}$, where $\eta_{i} \in\left\{ \pm \eta_{1}\right\}, \varepsilon_{i} \in\left\{ \pm \varepsilon_{1}\right\}$. Due to $\operatorname{dim} \mathfrak{S}(A+$ $B)=\operatorname{dim}(5(A-B)$, we see by Lemma 11 that

$$
\begin{aligned}
\operatorname{dim} \mathfrak{E}(\phi(A)+\phi(B)) & =\operatorname{dim} \mathfrak{\mathfrak { S }}(\phi(A)-\phi(B)) \\
& =\operatorname{dim} \mathfrak{\mathfrak { S }}(A-B)=\operatorname{dim} \mathfrak{\mathfrak { A }}_{n-4}+4 .
\end{aligned}
$$

Case 1. $n=5$. We first prove that $\operatorname{rank}(\phi(A) \pm \phi(B)) \neq 2$.

If $\operatorname{rank}(\phi(A)+\phi(B))=2$, then we may as well assume that $\phi(A)=\varepsilon P(K \oplus K \oplus 0) P^{t}$ and $\phi(B)=\varepsilon P(K \oplus-K \oplus 0) P^{t}$. Let $E=K \otimes I_{2} \oplus 0, F=K \otimes J \oplus 0$. It is easy to see that $[E, F]=0$. Now, we want to show that $[\phi(E), \phi(F)] \neq 0$, which is a contradiction. Note the following:

$$
\begin{array}{ll}
{[A,[A, E]]=-E,} & {[A,[A, F]]=-F,} \\
{[B,[B, E]]=-E,} & {[B,[B, F]]=-F .}
\end{array}
$$

So, we know that both $\phi(E)$ and $\phi(F)$ satisfy an equation about the matrix $X=\left[x_{i j}\right] \in \mathfrak{A}_{5}$ as follows:

$$
\begin{gathered}
{[\phi(A),[\phi(A), X]]=-X,} \\
{[\phi(B),[\phi(B), X]]=-X .}
\end{gathered}
$$

That is,

$$
\left[\begin{array}{ccccc}
0 & 0 & 2 \varepsilon^{2}\left(x_{24}-x_{13}\right) & -2 \varepsilon^{2}\left(x_{14}+x_{23}\right) & -\varepsilon^{2} x_{15} \\
0 & 0 & -2 \varepsilon^{2}\left(x_{14}+x_{23}\right) & 2 \varepsilon^{2}\left(x_{13}-x_{24}\right) & -\varepsilon^{2} x_{25} \\
2 \varepsilon^{2}\left(x_{13}-x_{24}\right) & 2 \varepsilon^{2}\left(x_{14}+x_{23}\right) & 0 & 0 & -\varepsilon^{2} x_{35} \\
2 \varepsilon^{2}\left(x_{14}+x_{23}\right) & 2 \varepsilon^{2}\left(x_{24}-x_{13}\right) & 0 & 0 & -\varepsilon^{2} x_{45} \\
\varepsilon^{2} x_{15} & \varepsilon^{2} x_{25} & \varepsilon^{2} x_{35} & \varepsilon^{2} x_{45} & 0
\end{array}\right]=-X
$$




$$
\left[\begin{array}{ccccc}
0 & 0 & -2 \varepsilon^{2}\left(x_{24}+x_{13}\right) & 2 \varepsilon^{2}\left(x_{23}-x_{14}\right) & -\varepsilon^{2} x_{15} \\
0 & 0 & 2 \varepsilon^{2}\left(x_{14}-x_{23}\right) & -2 \varepsilon^{2}\left(x_{13}+x_{24}\right) & -\varepsilon^{2} x_{25} \\
2 \varepsilon^{2}\left(x_{24}+x_{13}\right) & 2 \varepsilon^{2}\left(x_{23}-x_{14}\right) & 0 & 0 & -\varepsilon^{2} x_{35} \\
2 \varepsilon^{2}\left(x_{14}-x_{23}\right) & 2 \varepsilon^{2}\left(x_{13}+x_{24}\right) & 0 & 0 & -\varepsilon^{2} x_{45} \\
\varepsilon^{2} x_{15} & \varepsilon^{2} x_{25} & \varepsilon^{2} x_{35} & \varepsilon^{2} x_{45} & 0
\end{array}\right]=-X .
$$

Hence, we get that

$$
X=\left[\begin{array}{ccccc}
0 & 0 & 0 & 0 & x_{15} \\
0 & 0 & 0 & 0 & x_{25} \\
0 & 0 & 0 & 0 & x_{35} \\
0 & 0 & 0 & 0 & x_{45} \\
-x_{15} & -x_{25} & -x_{35} & -x_{45} & 0
\end{array}\right]
$$

and $\varepsilon^{2}=1$. Note that $[A+B, E]=0,[A-B, F]=0$. After taking the image, we can assume by $\varepsilon \neq 0$ that

$$
\begin{gathered}
\phi(E)=\left[\begin{array}{ccccc}
0 & 0 & 0 & 0 & 0 \\
0 & 0 & 0 & 0 & 0 \\
0 & 0 & 0 & 0 & a \\
0 & 0 & 0 & 0 & b \\
0 & 0 & -a & -b & 0
\end{array}\right], \\
\phi(F)=\left[\begin{array}{ccccc}
0 & 0 & 0 & 0 & c \\
0 & 0 & 0 & 0 & d \\
0 & 0 & 0 & 0 & 0 \\
0 & 0 & 0 & 0 & 0 \\
-c & -d & 0 & 0 & 0
\end{array}\right] .
\end{gathered}
$$

Again by $[E, F]=0$, we see that

$$
[\phi(E), \phi(F)]=\left[\begin{array}{ccccc}
0 & 0 & a c & b c & 0 \\
0 & 0 & a d & b d & 0 \\
-a c & -a d & 0 & 0 & 0 \\
-b c & -b d & 0 & 0 & 0 \\
0 & 0 & 0 & 0 & 0
\end{array}\right]=0 .
$$

We deduce that $a c=0, b c=0$. It follows by $\phi(E) \neq 0$ that $c=0$. Due to $a d=0, b d=0$, one has $d=0$. This tells us that $\phi(F)=0$, which is a contradiction. Similarly, we know that $\operatorname{rank}(\phi(A)-\phi(B)) \neq 2$.

Since $n=5$, it is clear that $\operatorname{rank}(\phi(A) \pm \phi(B))=4$. When

$$
\begin{gathered}
\phi(A)=\varepsilon P(K \oplus K \oplus 0) P^{t}, \\
\phi(B)=\eta P(K \oplus-K \oplus 0) P^{t},
\end{gathered}
$$

we have that $\varepsilon \neq \eta$. Note that $A \pm B$ is not a regular matrix; hence, $\phi(A) \pm \phi(B)$ is not too. Further, one has $\varepsilon+\eta \in\{ \pm(\varepsilon-$ $\eta)\}$. This implies that $\varepsilon=0$ or $\eta=0$, which is impossible. Similarly, we deduce that

$$
\begin{gathered}
\phi(A)=\varepsilon P(K \oplus-K \oplus 0) P^{t}, \\
\phi(B)=\eta P(K \oplus K \oplus 0) P^{t},
\end{gathered}
$$

which is also a contradiction.
Case 2. $n=6$, 8. We first prove that $\operatorname{rank}(\phi(A) \pm \phi(B)) \neq 2$. Otherwise, if $\operatorname{rank}(\phi(A)+\phi(B))=2$, then we have

$$
\operatorname{dim} \mathfrak{C}(\phi(A)+\phi(B))=\operatorname{dim} \mathfrak{A}_{n-2}+1 \neq \operatorname{dim} \mathfrak{A}_{n-4}+4,
$$

which is a contradiction. In a similar way, we get $\operatorname{rank}(\phi(A)-$ $\phi(B)) \neq 2$.

If $n=6$, we assert that $\operatorname{rank}(\phi(A) \pm \phi(B)) \neq 4$. In fact, if $\operatorname{rank}(\phi(A)+\phi(B))=4$, then by $\eta_{i} \in\left\{ \pm \eta_{1}\right\}, \varepsilon_{i} \in\left\{ \pm \varepsilon_{1}\right\}$, we deduce that $\eta_{i} \in\left\{ \pm \varepsilon_{1}\right\}$. Without loss of the generality, we can assume that $\varepsilon_{i}=\eta_{i}, i=1,2$, and $\varepsilon_{j}=-\eta_{j}, j=3$. Hence, we see that $\operatorname{rank}(\phi(A)-\phi(B))=2$, which is impossible. Similarly, we deduce that $\operatorname{rank}(\phi(A)-\phi(B)) \neq 4$.

Next, we prove when $n=6$ that $\operatorname{rank}(\phi(A) \pm \phi(B)) \neq 6$. Otherwise, by (64) we can assume without loss of the generality that $\varepsilon_{2}+\eta_{2} \in\left\{ \pm\left(\varepsilon_{1}+\eta_{1}\right)\right\}$ and $\varepsilon_{3}+\eta_{3} \notin\left\{ \pm\left(\varepsilon_{1}+\eta_{1}\right)\right\}$. Note that $\eta_{i} \in\left\{ \pm \eta_{1}\right\}, \varepsilon_{i} \in\left\{ \pm \varepsilon_{1}\right\}$, so we have $\varepsilon_{2}-\eta_{2} \in\left\{ \pm\left(\varepsilon_{1}-\eta_{1}\right)\right\}$ and $\varepsilon_{3}-\eta_{3} \notin\left\{ \pm\left(\varepsilon_{1}-\eta_{1}\right)\right\}$. Thus,

$$
\mathfrak{c}(\phi(A+B))=\mathfrak{c}(\phi(A-B)) .
$$

But it is clear that $\mathfrak{C}(A+B) \neq \mathfrak{C}(A-B)$, which contradicts with $\mathfrak{C}(\phi(X))=\phi(\mathfrak{C}(X))$, for all $X \in \mathfrak{A}_{n}$.

Similarly, we have when $n=8$ that $\operatorname{rank}(\phi(A) \pm$ $\phi(B)) \neq 6,8$.

Finally, we prove when $n=8$ that $\operatorname{rank}(\phi(A) \pm \phi(B)) \neq 4$. Let $Z=Q\left(E_{33}^{(4)} \otimes K\right) Q^{t}$. If rank $\phi(Z)=2$, then we can find a contradiction similar to the case of rank $\phi(B)=2$. Otherwise, if rank $\phi(Z)=8$, then there is an orthogonal matrix $P$ such that

$$
\begin{aligned}
\phi(A) & =P\left(\varepsilon_{1} K \oplus \cdots \oplus \varepsilon_{d} K\right) P^{t}, \\
\phi(B) & =P\left(\eta_{1} K \oplus \cdots \oplus \eta_{d} K\right) P^{t}, \\
\phi(Z) & =P\left(\lambda_{1} K \oplus \cdots \oplus \lambda_{d} K\right) P^{t},
\end{aligned}
$$

where $\eta_{i} \in\left\{ \pm \eta_{1}\right\}, \varepsilon_{i} \in\left\{ \pm \varepsilon_{1}\right\}$, and $\lambda_{i} \in\left\{ \pm \lambda_{1}\right\}$. It is easy to see that the three cases $\operatorname{rank}(\phi(A) \pm \phi(B))=4, \operatorname{rank}(\phi(A) \pm$ $\phi(Z))=4$, and $\operatorname{rank}(\phi(Z) \pm \phi(B))=4$ cannot simultaneously hold. This means that $\operatorname{rank}(\phi(A) \pm \phi(B))=4$ is impossible.

To sum up the previous arguments, we get that $\operatorname{rank} \phi(A)=2$. The proof is completed.

\section{Acknowledgments}

This paper is dedicated to Professor Chongguang Cao for his good health. The authors wish to thank Professor Xiaomin 
Tang for introducing and guiding the topic. The authors would also like to thank the referee for his/her careful reading of the paper and valuable comments which greatly improved the readability of the paper. Jinli $\mathrm{Xu}$ is supported in part by NSFC(11171294), Natural Science Foundation of Heilongjiang Province of China (Grant no. A201013). Baodong Zheng is supported by the National Natural Science Foundation Grants of China (Grant no. 10871056).

\section{References}

[1] G. Dolinar, "Maps on upper triangular matrices preserving Lie products," Linear and Multilinear Algebra, vol. 55, no. 2, pp. 191198, 2007.

[2] F. Kuzucuoğlu, "Isomorphisms of the unitriangular groups and associated Lie rings for the exceptional dimensions," Acta Applicandae Mathematicae, vol. 85, no. 1-3, pp. 209-213, 2005.

[3] P. S. Blau, "Lie isomorphisms of prime rings satisfying $S t_{4}$," Southeast Asian Bulletin of Mathematics, vol. 25, no. 4, pp. 581587, 2002.

[4] D. Wang, Y. Zhao, and Z. Chen, "Nonlinear maps on simple Lie algebras preserving Lie products," Communications in Algebra, vol. 39, no. 2, pp. 424-434, 2011.

[5] X.-M. Tang and J.-L. Xu, "The derivation Lie algebra of the higher rank Virasoro-like algebra and its automorphism groups," Linear Algebra and Its Applications, vol. 430, no. 8-9, pp. 2170-2181, 2009.

[6] C. Park and A. Najati, "Homomorphisms and derivations in $C^{*}$ algebras," Abstract and Applied Analysis, vol. 2007, Article ID 80630, 12 pages, 2007.

[7] C. Park, J. S. An, and J. Cui, "Isomorphisms and derivations in Lie $C^{*}$-algebras," Abstract and Applied Analysis, vol. 2007, Article ID 85737, 14 pages, 2007.

[8] L.-K. Hua, "A theorem on matrices over a sfield and its applications," Acta Mathematica Sinica, vol. 1, pp. 110-163, 1951.

[9] N. Jacobson, Lie Algebra, Interscience Publishers, New York, NY, USA, 1962.

[10] M. Brešar, M. A. Chebotar, and W. S. Martindale III, Functional Identities, Frontiers in Mathematics, Birkhäuser, Basel, Switzerland, 2007.

[11] W. S. Martindale III, "Lie isomorphisms of the skew elements of a prime ring with involution," Communications in Algebra, vol. 4, no. 10, pp. 929-977, 1976.

[12] R. A. Horn and C. R. Johnson, Matrix Analysis, Cambridge University Press, Cambridge, UK, 1985.

[13] C. Cao, L. Huang, and X. Tang, "Additive map preserving rank 2 on alternate matrices," African Diaspora Journal of Mathematics, vol. 3, no. 2, pp. 107-113, 2005.

[14] X. Zhang, "Linear/additive preservers of rank 2 on spaces of alternate matrices over fields," Linear Algebra and Its Applications, vol. 396, pp. 91-102, 2005. 


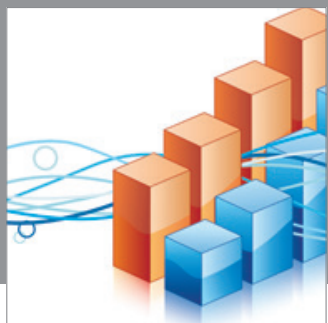

Advances in

Operations Research

mansans

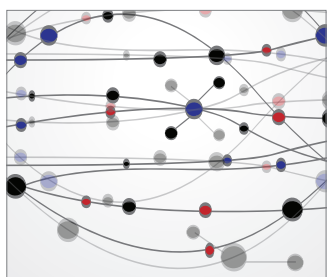

The Scientific World Journal
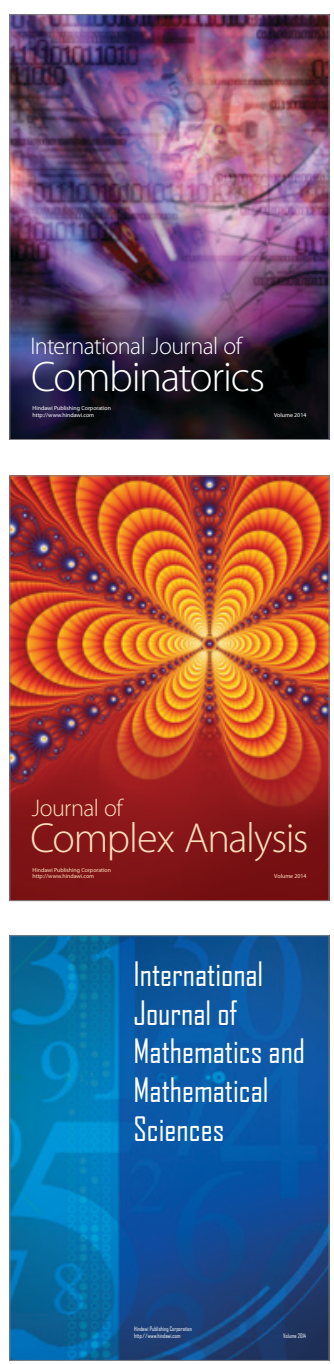
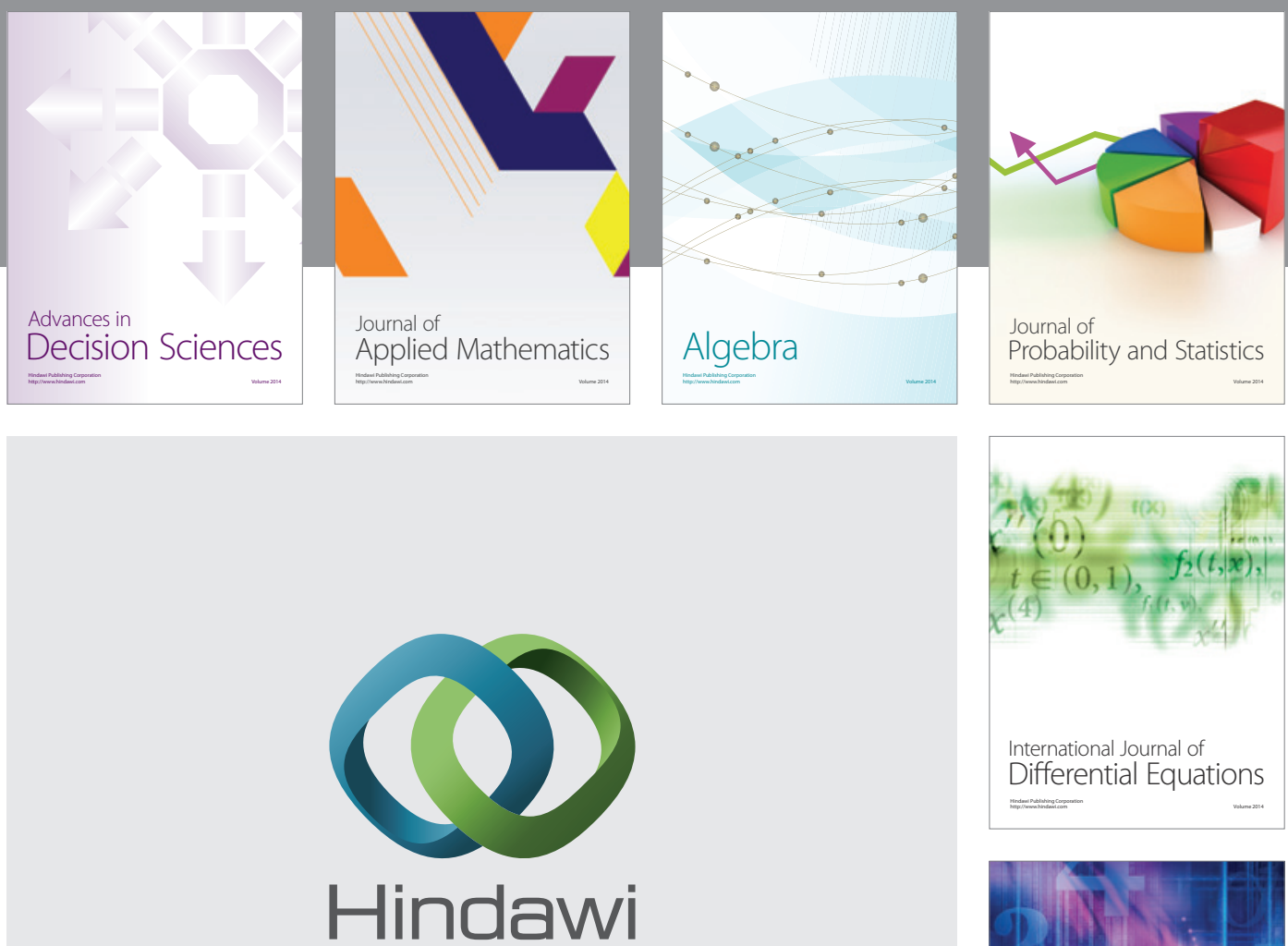

Submit your manuscripts at http://www.hindawi.com
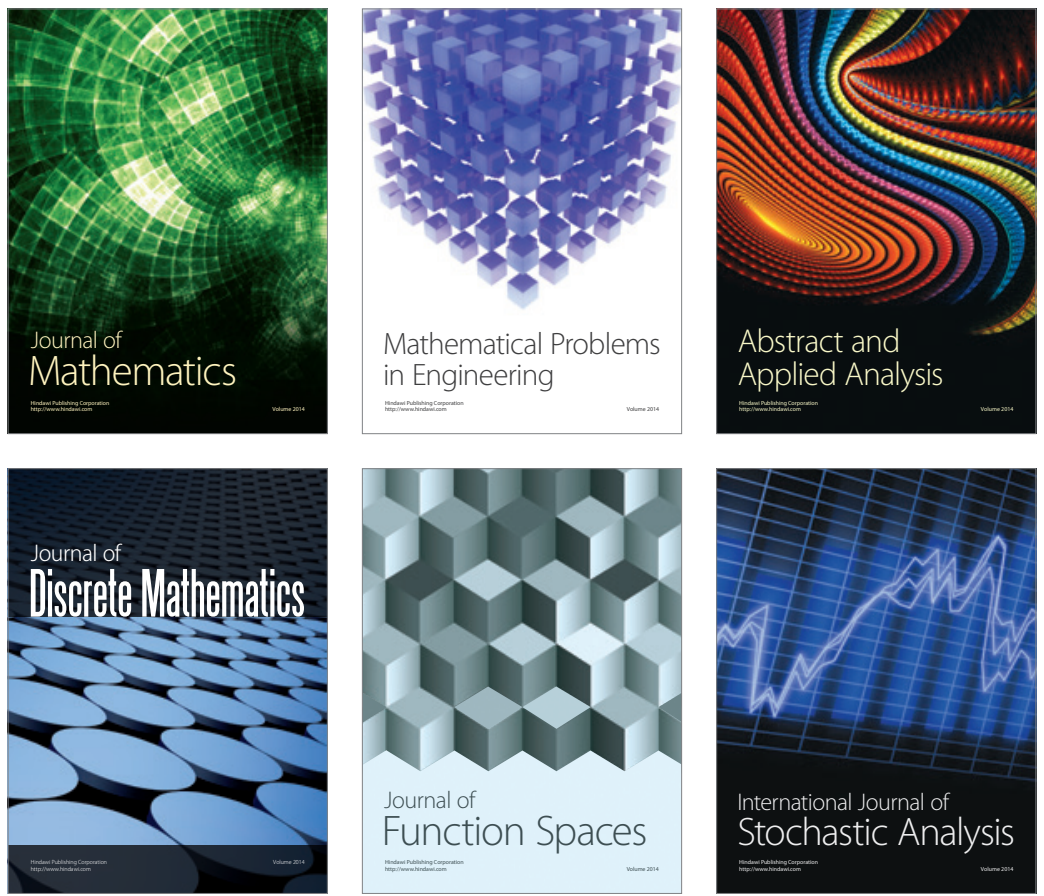

Journal of

Function Spaces

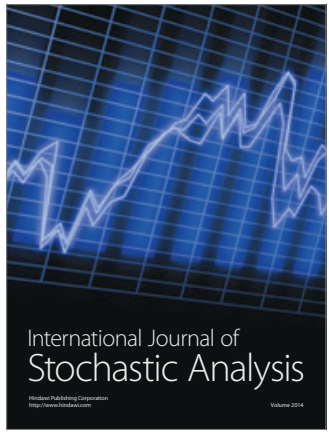

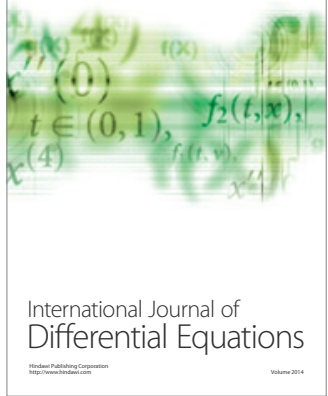
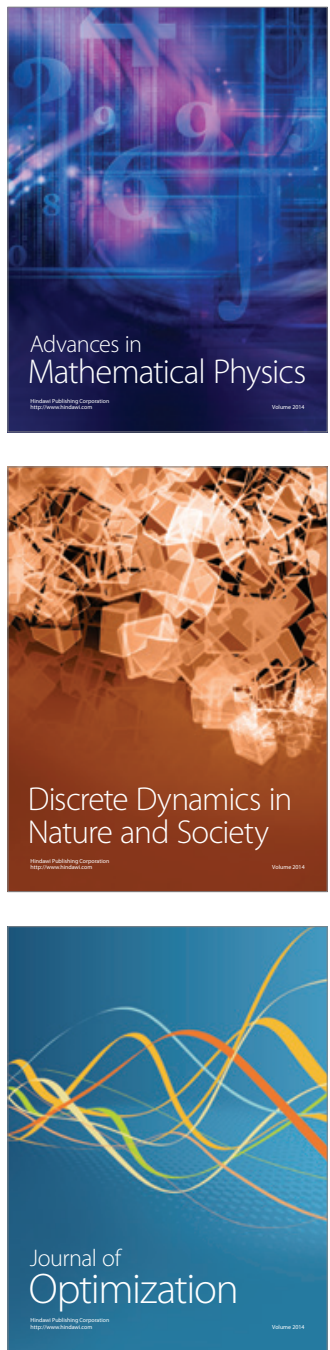